\title{
Large-Grain Polycrystalline Silicon Solar Cell on Epitaxial Thickening of AIC Seed Layer by Hot Wire CVD
}

\author{
Jui-Hao Wang, Shui-Yang Lien, Chia-Fu Chen, and Wha-Tzong Whang
}

\begin{abstract}
Large-grain polycrystalline silicon (poly-Si) films were prepared on foreign substrates by the epitaxial thickening of seed layers. The seed layers were formed by aluminum-induced crystallization (AIC). Large-grain n-i-p poly-Si solar cells were deposited on epitaxial seeds by hot-wire chemical vapor deposition (HWCVD). Highly (93\%) crystalline fractions with a lateral grain size of $5 \mu \mathrm{m}$ and an intrinsic layer were grown without incubation. These techniques were employed to prepare largegrain poly-Si thin-film solar cells. An ITO/n-i-p (HWCVD)/p+ (AIC)/Ti/glass-structured poly-Si thin-film solar cell with an initial efficiency of $5.6 \%$ was obtained.
\end{abstract}

Index Terms-Aluminum-induced crystallization (AIC), hot-wire chemical vapor deposition (HWCVD), polycrystalline silicon (poly-Si).

\section{INTRODUCTION}

$\mathbf{T}$ HE FORMATION of high-quality polycrystalline silicon (poly-Si) on low-cost substrates has important applications in the development of electrical devices. Metal-induced crystallization is a promising method for the low-temperature fabrication of large-area poly-Si as a seeding layer for electron device applications [1]-[3]. One of the factors that determine the suitability of any deposition process for particular device applications is the deposition rate. Material researchers face the challenge of maintaining device quality while increasing the deposition rate. Hot-wire chemical vapor deposition (HWCVD) is a popular approach for producing amorphous silicon (a-Si) and poly-Si material at a high deposition rate [4], [5]. The a-Si films of device quality can be obtained by HWCVD using pure silane $\left(\mathrm{SiH}_{4}\right)$ without hydrogen dilution. Poly-Si films of high quality are obtained when silane is diluted in hydrogen at high pressure. However, the deposition-rate decreases as the hydrogen dilution increases. The direct deposition of poly-Si films is an inhomogeneous growth process [6]. The deposition generally begins with the formation of an amorphous phase,

Manuscript received September 17, 2009. First published November 20, 2009; current version published December 23, 2009. This work was supported by the National Science Council of the Republic of China under Contract NSC 97-2221-E-451-014. The review of this letter was arranged by Editor C. Jagadish.

J.-H. Wang and W.-T. Whang are with the Department of Materials Science and Engineering, National Chiao Tung University, Hsinchu 300, Taiwan (e-mail: tab133kimo749@msn.com).

S.-Y. Lien and C.-F. Chen are with the Department of Materials Science and Engineering, MingDao University, Changhua 52345, Taiwan (e-mail: syl@mdu.edu.tw).

Color versions of one or more of the figures in this letter are available online at http://ieeexplore.ieee.org.

Digital Object Identifier 10.1109/LED.2009.2035141 which, when its thickness exceeds a minimum thickness, is called the incubation phase, which is present before a localized phase transformation, called nucleation, takes place. In this letter, to make polycrystalline films at high deposition rates without an incubation phase, a seed-layer deposition process is applied. $\mathrm{p}^{+}$-type poly-Si films are prepared by AIC on glass substrate. This is epitaxially thickened. This letter focuses on the properties of poly-Si deposited on a seed layer at a very high deposition rate $(5 \mathrm{~nm} / \mathrm{s})$. Finally, the performance of poly-Si solar cell with seed layer is compared with poly-Si solar cell without seed layer.

\section{EXPERIMENTS}

In this letter, the $\mathrm{p}^{+}$-type seed layers were prepared by AIC technology. The Ti and Al layers $(200 \mathrm{~nm})$ were deposited on glass substrate (Corning 1737) by EB evaporation and then a Si layer $(200 \mathrm{~nm})$ was deposited by HWCVD. Thermal annealing was performed in a conventional furnace at $450{ }^{\circ} \mathrm{C}$ for $5 \mathrm{~h}$. After the AIC process, the $\mathrm{Al}(+\mathrm{Si})$ layer on top of the seed layer had to be removed. In this study, two main types of poly-Si are formed: one with columnar crystals (called Poly1) and the other with V-shaped crystals (called Poly2). Poly1 contains seed layers that are produced by the AIC process. Poly2 $(1200 \mathrm{~nm})$ is deposited using a low hydrogen dilution of silane source gas, at a much higher deposition rate of $5 \mathrm{~nm} / \mathrm{s}$. The hydrogen dilution is defined as $\Phi \mathrm{H}_{2} /\left(\Phi \mathrm{SiH}_{4}+\Phi \mathrm{H}_{2}\right)$. The $\Phi \mathrm{SiH}_{4}$ and $\Phi \mathrm{H}_{2}$ are $\mathrm{SiH}_{4}$ and $\mathrm{H}_{2}$ gas flow rates, respectively. Crystalline fraction $\left(R_{c}\right)$ was evaluated by Raman-scattering spectroscopy [7]. The $R_{c}$ of the material was calculated as $R_{c}=\left(I_{520}+I_{510}\right) /\left(I_{520}+I_{510}+I_{480}\right)$, where $I_{x}$ is the integrated Gaussian that corresponds to mode $x$ in the Raman spectrum. The microstructures of Poly2 (HWCVD)/Poly1 (AIC)/glass are observed by field-emission scanning electron microscopy and transmission electron microscopy (TEM). Current density-voltage $(J-V)$ measurements were made under AM1.5G $\left(100 \mathrm{~mW} / \mathrm{cm}^{2}\right)$ white light from a dual-beam solar simulator.

\section{RESULTS AND DisCUSSION}

Fig. 1 shows the Raman spectra of the Si films prepared using different growth techniques. From the Raman results, lines (a) and (b) correspond to the a-Si and poly-Si films that were deposited directly by HWCVD on the glass. They were deposited using low hydrogen dilution (30\%) and high hydrogen dilution $(98 \%)$, respectively; revealing that increasing 


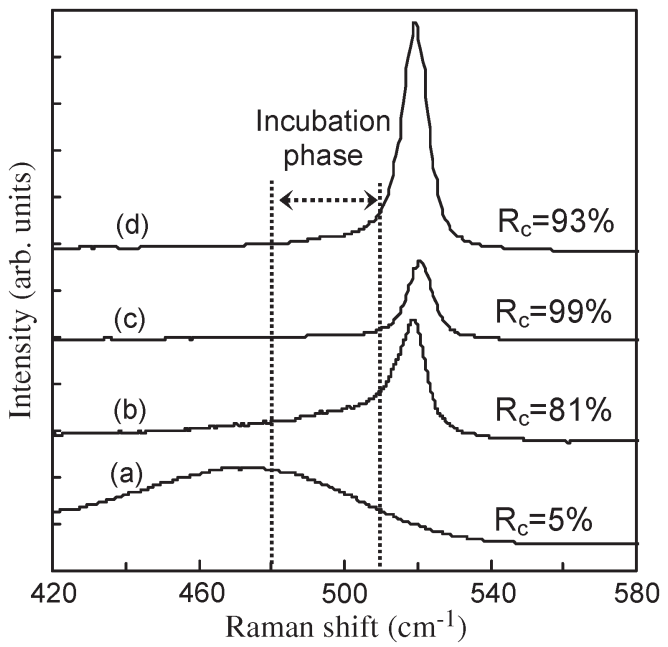

Fig. 1. Raman spectra of samples. (a) a-Si films deposited by HWCVD. (b) poly-Si films deposited by HWCVD. (c) AIC poly-Si films. (d) Poly-Si (HWCVD)/Poly-Si (AIC)/glass structure.

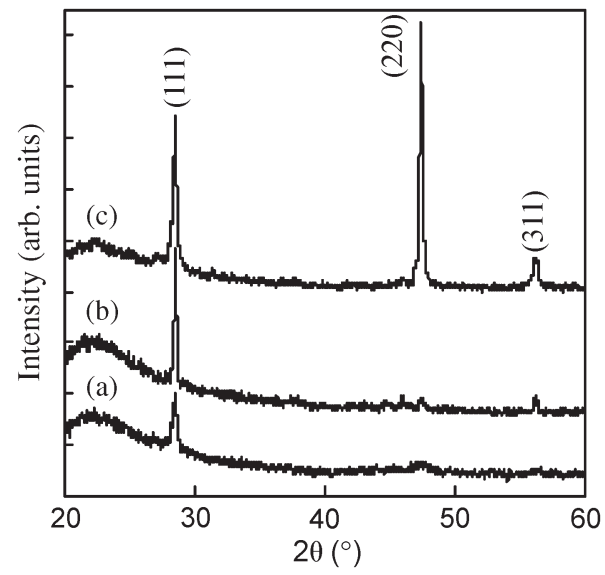

Fig. 2. XRD patterns of diffraction samples. (a) a-Si films deposited by HWCVD. (b) AIC poly-Si films after annealing and etching. (c) Glass/ poly-Si (AIC)/poly-Si (HWCVD).

the hydrogen dilution increases the crystallinity of the material. Line (c) shows the properties of the poly-Si film produced by the AIC process. This material does not include any incubation phase (Raman shift between 480 and $510 \mathrm{~cm}^{-1}$ ). Line (d) demonstrates that the use of a seed layer, formed by AIC, enables highly crystalline layers to be deposited by HWCVD using a hydrogen dilution of less than $30 \%$. A poly $2 /$ poly 1 thin film with a high crystalline fraction of $93 \%$ was observed. When no seed layer was used, this lower hydrogen dilution yielded amorphous layers.

Fig. 2 shows the X-ray diffraction (XRD) spectra of the poly-Si layers that are produced using various technologies.

The XRD measurement [line (a)] was made on one set of a-Si samples that were deposited by HWCVD using low hydrogen dilution (30\%). Line (b) shows that the poly-Si was formed by AIC with strong (111) and weak (220) orientations. However, poly-Si films made by HWCVD (Poly2) at a low hydrogen dilution were grown along the AIC grains. The spectrum obtained after the $\mathrm{Al}$ film was removed has no $\mathrm{Al}$ peak. Nevertheless, line (c) shows that the poly-Si was deposited on AIC poly-Si films by HWCVD with highly scattered (111) and (220) and weakly

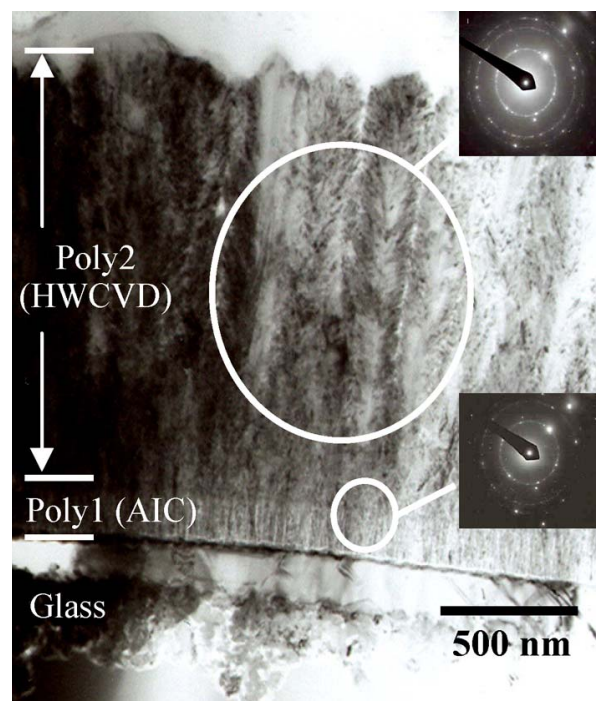

Fig. 3. TEM cross-sectional image of poly2 (HWCVD)/poly1 (AIC)/glass and its diffraction pattern.

scattered (311) peaks. This poly-Si was formed at a temperature as low as $250^{\circ} \mathrm{C}$ and a deposition rate as high as $5 \mathrm{~nm} / \mathrm{s}$.

Fig. 3 shows a TEM cross-sectional image of Poly2 $(1200 \mathrm{~nm}) /$ Poly1 (200 nm)/glass. Fig. 3 clearly displays a nonintentionally doped Si film grown by HWCVD on a glass/AIC seed-layer sample. Poly-Si films were formed with a compact structure of columnar grains by the AIC technique (Poly1). The diffraction patterns (inset below) show the polycrystalline structure of the sample. The diffraction rings correspond to (111) and (220) preferential orientations. Diffraction rings with clear diffraction spots, indicating a highly ordered structure are observed, implying that each grain in this region consists of crystalline phases of mainly a single domain. HWCVD poly-Si films were fully polycrystalline, with $\mathrm{V}$-shaped grains. The lateral grain size of the poly-Si films deposited on the seed layer was approximately $5 \mu \mathrm{m}$ higher than that of the poly-Si films that were deposited directly on the glass by HWCVD with high hydrogen dilution. The diffraction patterns (inset above) show the polycrystalline structure of the sample. The diffraction rings correspond to (111), (220), and (311) preferential orientations. The ring patterns are dominant and include some spots, revealing that the film includes single crystals. Thus, the crystalline Si film was grown on an epitaxial-thickened AIC seed layer, eliminating the need for an amorphous incubation layer and the deposition of poly-Si films, avoiding the formation of any amorphous phase.

Fig. 4 shows the TEM image of poly2 (HWCVD)/poly1 (AIC)/glass structure and its diffraction pattern. This is the TEM of the poly2 (HWCVD) part of that sample, rather than the whole sample that was shown in Fig. 3. The lateral grain size of the poly 2 films that were deposited on the poly1 layer was approximately $5 \mu \mathrm{m}$. Although the individual crystallites are quite large, they are very irregular in shape. The diffraction patterns (inset below) show the polycrystalline structure of the sample. Halo patterns that correspond to amorphous silicon are not observed, revealing that the layer is completely crystallized. The convergent-beam electron diffraction pattern (inset above) shows clear diffraction spots, which reveal a highly ordered 


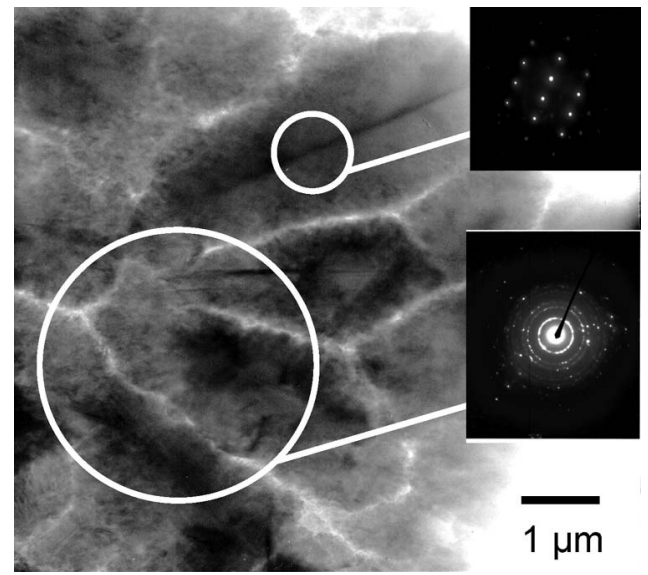

Fig. 4. TEM plane view of poly2 (HWCVD)/poly1 (AIC)/glass structure and its diffraction pattern.

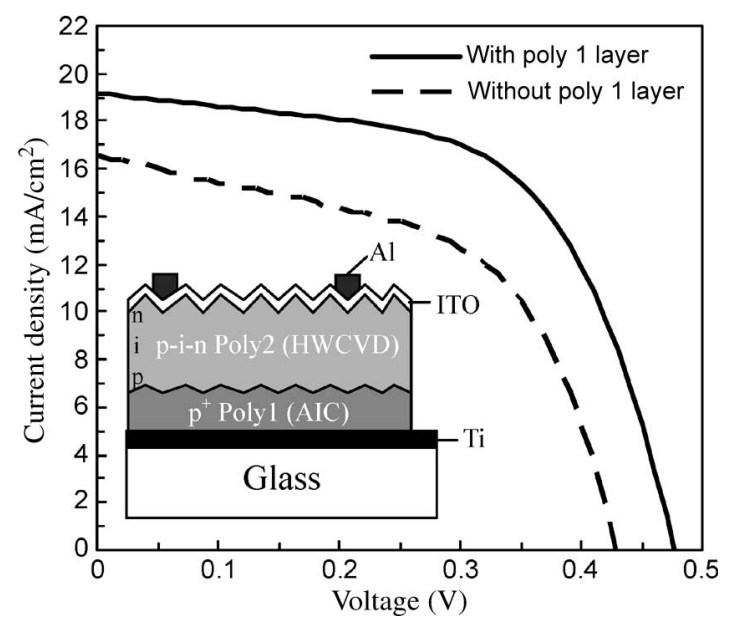

Fig. 5. Current-voltage characteristic of the poly-Si thin-film solar cells with poly1 and without poly1 layer.

structure. Hence, each grain in this region consists of crystalline phases of a mainly single domain.

Fig. 5 shows the current density-voltage $(J-V)$ characteristic of the poly-Si solar cell with poly1 and without poly1 layer. The inset in Fig. 5 schematically shows the structure of poly-Si thin-film solar cells. The performance of the poly-Si solar cells with and without poly1 layer in the presented work follows from the stable HWCVD, as shown in Table I. The cell with poly1 layer has a fill factor of 0.63 , an open-circuit voltage of $0.475 \mathrm{~V}$, a short-circuit current density of $19 \mathrm{~mA} / \mathrm{cm}^{2}$, and an initial efficiency of 5.6\%. The large-grain poly-Si films were produced by AIC (poly1) and HWCVD (poly2) techniques.
TABLE I

Performance of Solar Cell With and Without Poly1 Layer

\begin{tabular}{lcc}
\hline Performance & With poly1 layer & Without poly1 layer \\
\hline $\mathrm{V}_{\mathrm{oc}}(\mathrm{V})$ & 0.47 & 0.43 \\
$\mathrm{~J}_{\mathrm{sc}}\left(\mathrm{mA} / \mathrm{cm}^{2}\right)$ & 19.1 & 16.6 \\
$\mathrm{FF}$ & 0.62 & 0.54 \\
$\eta(\%)$ & 5.6 & 3.8 \\
\hline
\end{tabular}

A highly efficient poly-Si thin-film solar cell with an Ag/ITO/ $\mathrm{n}-\mathrm{i}-\mathrm{p} / \mathrm{AIC}(\mathrm{p}+) / \mathrm{Ti}$ structure on a glass substrate was obtained.

\section{CONCLUSION}

Large-grain poly-Si films were obtained by HWCVD at a high deposition rate $(5 \mathrm{~nm} / \mathrm{s})$ on epitaxially thickened AIC seed layers. Highly crystalline fractions with $93 \%$ crystallinity, a lateral grain size of $5 \mu \mathrm{m}$, and an intrinsic layer that was grown without incubation, were obtained in this investigation. This work elucidates a procedure for poly-Si films that eliminates an incubation phase. Large-grain poly-Si solar cells were produced by AIC and HWCVD techniques. An Ag/ITO/ $\mathrm{n}$-i-p/AIC $(\mathrm{p}+) / \mathrm{Ti}$-structured solar cell on glass structure with an initial efficiency of $5.6 \%$ was obtained.

\section{ACKNOWLEDGMENT}

The authors would like to thank T. Knoy for his editorial assistance.

\section{REFERENCES}

[1] O. Nast, S. Brehme, D. H. Neuhaus, and S. R. Wenham, "Polycrystalline silicon thin films on glass by aluminum-induced crystallization," IEEE Trans. Electron Devices, vol. 46, no. 10, pp. 2062-2068, Oct. 1999.

[2] S. Y. Yoon, N. Young, P. J. van der Zaag, and D. McCulloch, "Highperformance poly-Si TFTs made by Ni-mediated crystallization through low-shot laser annealing," IEEE Electron Device Lett., vol. 24, no. 1, pp. 22-24, Jan. 2003.

[3] J. H. Cheon, J. H. Bae, and J. Jang, "Inverted staggered poly-Si thin-film transistor with planarized SOG gate insulator," IEEE Electron Device Lett., vol. 29, no. 3, pp. 235-237, Mar. 2008.

[4] A. H. Mahan, J. Carapella, B. P. Nelson, R. S. Crandall, and I. Balberg, "Deposition of device quality, low $\mathrm{H}$ content amorphous silicon," J. Appl. Phys., vol. 69, no. 9, pp. 6728-6730, May 1991.

[5] H. Matsumura, "Catalytic chemical vapor deposition (CAD-CVD) method producing high quality hydrogenated amorphous silicon," Jpn. J. Appl. Phys., vol. 25, pp. L949-L951, 1986.

[6] D. S. Wuu, S. Y. Lien, H. Y. Mao, B. R. Wu, I. C. Hsieh, P. C. Yao, J. H. Wang, and W. C. Chen, "Growth and characterization of polycrystalline Si films prepared by hot-wire chemical vapor deposition," Thin Solid Films, vol. 498, no. 1/2, pp. 9-13, Mar. 2006.

[7] Z. Iqbal, S. Veprek, A. P. Webb, and P. Capezzuto, "Raman scattering from small particle size polycrystalline silicon," Solid State Commun., vol. 37, no. 12, pp. 993-996, Mar. 1981. 